\title{
И.А. ИЛЬИН
}

\section{РЕЗУЛЬТАТЫ ПОВТОРНОЙ ТОЛСТОКИШЕЧНОЙ ПЛАСТИКИ ПИЩЕВОДА ПРИ ХИРУРГИЧЕСКОМ ЛЕЧЕНИИ КАРЦИНОМ ПИЩЕВОДА И ПИЩЕВОДНО-ЖЕЛУДОЧНОГО ПЕРЕХОДА}

\author{
Республиканский научно-практический центр онкологии \\ и медицинской радиологии им. Н.Н. Александрова, г. Минск, \\ Республика Беларусь
}

\begin{abstract}
Цель. Оценить результаты повторной толстокишечной пластики пищевода при хирургическом лечении карцином пищевода и пищеводно-желудочного перехода.

Материал и методы. Повторные загрудинные толстокишечные пластики пищевода после разобщающих операций по поводу осложнений первичной реконструкции пищевода желудком или тонкой кишкой при хирургическом лечении карцином пищевода и пищеводно-желудочного перехода выполнены у 40 пациентов. Плоскоклеточный рак пищевода был представлен в 52,5\% (21/40) наблюдений и аденокарцинома пищеводно-желудочного перехода - в 47,5\% (19/40). Средний возраст пациентов составил 56,0 (52,0; $63,0)$ лет, индекс массы тела - 21,4 $(18,9 ; 24,5)$ Ед. Пациенты мужского пола преобладали над женским $90 \%(36 / 40)$ и $10 \%$ (4/40) человек соответственно. Повторным реконструкциям предшествовали ранее выполненные разобщающие операции по поводу осложнений первичной пластики пищевода желудком или тонкой кишкой. Резекция пищеводно-желудочного анастомоза выполнена у 57,5\% (23/40) пациентов, удаление гастротрансплантата - у 7,5\% (3/40), демукозация пищевода с резекцией осложненного анастомоза у $35 \%(14 / 40)$.

Результаты. Длительность вмешательств составила $345,0(310,0 ; 407,5)$ минут, объем кровопотери $400,0(300,0 ; 500,0)$ мл, продолжительность стационарного лечения - 27,5 $(21,0 ; 40,5)$ дня. Несостоятельность пищеводно-толстокишечного анастомоза и некроз проксимальных отделов трансплантата выявлялись клинически и рентгенологически на 8-е сутки после операции. Несостоятельность анастомоза развилась в $12,5 \%$ (5/40) наблюдений, некроз - в 5\% (2/40). Поздние рубцовые стриктуры пищеводно-толстокишечного анастомоза, диагностированные по данным фиброэзофагоколоноскопии спустя 3 месяца после повторной эзофагопластики, сформировались в 7,5\% (3/40) наблюдений. 30- и 60 -дневная летальность в группах составила $-7,5 \%(3 / 40)$ и $10 \%$ (4/40) случаев соответственно. Общая 5-летняя выживаемость пациентов равнялась $26,9 \%$.
\end{abstract}

Заключение. Повторная толстокишечная пластика пищевода при хирургическом лечении карцином пищевода и пищеводно-желудочного перехода является спасительной процедурой для пациентов, перенесших разобщающие (стомирующие) оперативные вмешательства, позволяющей добиться удовлетворительных отдаленных результатов лечения.

Ключевые слова: кариинома пищевода, карцинома пищеводно-жслудочного перехода, повторная толстокищечная пластика пищевода, загрудинный путь проведения трансплантата, разобщающая операция, демукозация пищевода

Objective. To evaluate the results of the repeated colon interposition for esophageal replacement in the esophageal and gastroesophageal junction carcinomas surgical treatment.

Methods. Repeated colon interposition after discontinuity resections for ischemic complications of primary gastric or jejunal pull up procedures during the esophageal and gastroesophageal junction carcinomas surgical treatment was implemented in 40 patients. Squamous cell esophageal carcinoma was represented in $52.5 \%(21 / 40)$ observations and gastroesophageal junction adenocarcinoma - in 47.5\% (19/40). Patients' mean age was 56.0 (52.0, 63.0) years, body mass index $-21.4(18.9,24.5)$ units. Male patients predominated over females $-90 \%(36 / 40)$ and $10 \%(4 / 40)$ respectively. Repeated reconstructions were preceded by previously performed discontinuity procedures. Esophago-gastric anastomosis resection was performed in 57.5\% (23/40) patients, gastric conduit removal in 7.5\% (3/40), esophageal demucosation with complicated anastomosis resection in $35 \%(14 / 40)$.

Results. Time of procedures was $345.0(310.0,407.5)$ minutes, blood loss - $400.0(300.0,500.0) \mathrm{ml}$, inhospital stay $-27.5(21.0,40.5)$ days. Esophago-colonic anastomosis leakage and/or an oral end graft necrosis were detected clinically and radiologically on the 8th day after surgery. Anastomotic leakage developed in $12.5 \%$ $(5 / 40)$ observations, graft loss - in 5\% (2/40). Late esophageal anastomotic strictures, determined by means of esophagocolonoscopy 3 months after the repeated esophagoplasty, were formed in 7.5\% (3/40) patients. 30- and 60-day mortality rate made up 7.5\% (3/40) and 10\% (4/40) cases respectively. Overall 5-year survival was $26.9 \%$.

Conclusions. Repeated colon interposition for esophageal replacement in esophageal and gastroesophageal junction carcinomas surgical treatment is a "salvage" procedure for patients who undergo discontinuity surgical interventions which allows achieving satisfactory long-term treatment outcomes. 
Keywords: esophageal carcinoma, gastroesophageal junction carcinoma, repeated esophageal replacement by colon interposition, retrosternal route of graft translocation, discontinuity procedure, esophageal demucosation

Novosti Khirurgii. 2019 Mar-Apr; Vol 27 (2): 177-187

The articles published under CC BY NC-ND license Results of Repeated Colon Interposition for Esophageal Replacement in Esophageal and Gastroesophageal Junction in Carcinoma Surgical Treatment I.A. Ilyin

\section{Научная новизна статьи}

Впервые проанализированы результаты повторной толстокишечной пластики пищевода у пациентов, перенесших разобщающие оперативные вмешательства по поводу ишемических осложнений после первичной пластики пищевода желудком или тонкой кишкой, при хирургическом лечении карцином пищевода и пищеводно-желудочного перехода. Установлено, что реконструкция пищевода у стомированных пациентов в условиях дефицита пластического материала позволяет добиться удовлетворительных отдаленных результатов лечения.

\section{What this paper adds}

The results of the repeated colonic interposition for esophageal replacement have been analyzed for the first time in patients who underwent discontinuity surgical procedures for ischemic complications after primary esophageal reconstruction by gastric or jejunal pull up during the esophageal and gastroesophageal junction carcinomas surgical treatment. It has been established that esophageal replacement in cases of plastic material deficiency allows achieving satisfactory long-term results of treatment.

\section{Введение}

Проблема повторной реконструкции пищевода у онкологических пациентов после разобщающих операций, выполненных по поводу ишемических осложнений (некроз трансплантата и/или несостоятельность анастомоза) первичной эзофагопластики при хирургическом лечении карцином пищевода и пищеводно-желудочного перехода, сохраняет свою актуальность и в настоящее время. Хирургия пищевода всегда связана с определенным риском развития осложнений со стороны пищеводно-органного анастомоза и висцерального трансплантата, использованного для эзофагопластики. При этом в ряде случаев возникает необходимость выполнения разобщения непрерывности пищеварительного тракта с целью устранения источника септического очага из средостения. Разобщающие операции сопровождаются инвалидизацией пациентов за счет стомирования-формирование эзофагостомы и гастро- или еюностомы, что требует принятия решения в отношении выполнения повторной пластики пищевода, где в роли наиболее подходящего пластического материала выступает собственная толстая кишка из-за нетрансплантабельности желудка и тонкой кишки.

Проблеме восстановления непрерывности пищеварительного тракта у стомированных пациентов после осложнений первичной пластики при хирургическом лечении рака верхних отделов пищеварительного тракта в имеющейся отечественной и зарубежной литературе отведено крайне мало места, а опыт представлен немногочисленными наблюдениями $[1,2,3,4,5]$.
Повторная эзофагопластика представляет собой крайне трудную проблему онкохирургии, нередко требующую применения нежелудочных вариантов реконструкции пищевода из имеющегося в наличии пластического резерва в виде ободочной кишки. Трудности пластики у данной категории пациентов обусловлены сниженными пластическими резервами, дефицитом массы тела, наличием сопутствующей патологии, спаечными процессами брюшной полости после предшествующих вмешательств, трудностями при мобилизации выбранного кишечного сегмента. Нередко при измененной сосудистой анатомии возникает необходимость создания источника дополнительного кровоснабжения для обеспечения эффективного кровотока в искусственном пищеводе [2, 6, 7, 8, 9].

Восстановление естественного пищеварительного пассажа у онкологических пациентов при данных обстоятельствах является единственно возможной «спасительной» процедурой в условиях дефицита пластического материала. Повторная пластика пищевода несет в себе потенциал долговременной выживаемости за счет непосредственного улучшения качества жизни пациентов и создания условий для дополнительного специального противоопухолевого лечения в случае прогрессирования карциномы пищевода или пищеводно-желудочного перехода.

Необходимость совершенствования хирургического метода лечения и повышения его эффективности определяет актуальность представленного материала.

Цель. Оценить результаты повторной толстокишечной пластики пищевода при хирургическом лечении карцином пищевода и пищеводно-желудочного перехода. 


\section{Материал и методы}

Повторная загрудинная толстокишечная пластика пищевода после разобщающих операций по поводу осложнений первичной реконструкции пищевода желудком или тонкой кишкой при хирургическом лечении карцином пищевода и пищеводно-желудочного перехода выполнена у 40 пациентов.

В структуре онкопатологии в общей когорте пациентов в равной мере встречался плоскоклеточный рак пищевода $-52,5 \%$ (21/40) и аденокарцинома пищеводно-желудочного перехода 47,5\% (19/40) $(\mathrm{p}=0,823)$. По фактору рТ пациенты распределились следующим образом: рТ1 7,5\% (3/40), pT2 - 25\% (10/40), pT3 - 47,5\% (19/40) пациентов, pТ4 - 20\% (8/40); по фактору pN: pN0 - 57,5\% (23/40), pN1-37,5\% (15/40), $\mathrm{pN} 2-5 \%$ (2/40) случаев; по стадиям: III стадия $50 \%$ (20/40), II стадия - 30\% (12/40), I стадия $17,5 \%$ (7/40), IV стадия - 2,5\% (1/40) наблюдений. Средний возраст пациентов составил 56,0 $(52,0 ; 63,0)$ лет, индекс массы тела - 21,4 $(18,9$; $24,5)$ Ед. Пациенты мужского пола доминировали над лицами женского пола - 90\% (36/40) и $10 \%(4 / 40)$ человек соответственно $(\mathrm{p}<0,001)$.

Использование толстой кишки в качестве пластического материала при создании искусственного пищевода было обусловлено нетрансплантабельностью по длине желудка и тонкой кишки для отдаленного перемещения на шею из-за предшествуюшей разобщающей операции. Так, резекция пищеводно-желудочного анастомоза в пределах здоровых тканей выполнена у 57,5\% (23/40) пациентов, удаление гастротрансплантата - у 7,5\% (3/40), демукозация пищевода с резекцией осложненного анастомоза - у 35\% (14/40).

В общей когорте преимущественно формировались трансплантаты в изоперистальтической позиции, сформированные путем субтотальной мобилизации толстой кишки, по сравнению с антиперистальтической позицией, при которой требовалась тотальная мобилизация ободочной кишки $-80 \%(32 / 40)$ и $20 \%(8 / 40)$ соответственно $(\mathrm{p}<0,001)$. При образовании трансплантата использовались как правый, так и левый фланги толстой кишки $-60 \%(24 / 40)$ и $40 \%(16 / 40)$ соответственно $(\mathrm{p}=0,117)$. В роли питающей сосудистой ножки чаще выступали левые ободочные сосуды по сравнению со средними - 65\% (26/40) против $30 \%(12 / 40)$ соответственно ( $=0,003)$. Двойного питания трансплантата за счет средних и левых ободочно-кишечных сосудов удалось достичь всего в нескольких наблюдениях по сравнению с другими вариантами - 5\% (2/40) против 95\%
$(38 / 40)$ соответственно $(\mathrm{p}<0,001)$.

Проксимальные анастомозы пищевода с толстокишечным трансплантатом накладывали по типу конец в конец в 65\% (26/40) случаев, конец в бок - в $30 \%(12 / 40)$. В $5 \%(2 / 40)$ наблюдений анастомоз был сформирован с глоткой. Дистальные дигестивные анастомозы с трансплантатом формировали следующим образом: конец в переднюю стенку желудка $27,5 \%(11 / 40)$, конец в бок тонкокишечной петли - 47,5\% (19/40), конец в бок на петле по Ру - 17,5\% (7/40), конец в переднюю стенку двенадцатиперстной кишки - 7,5\% (3/40) соответственно. При формировании 11 толстокишечно-желудочных анастомозов пилоропластика потребовалась только 27,3\% (3/11) пациентов.

Сосудистое усиление путем васкуляризации трансплантата применено у 42,5\% (17/40) пациентов. Роль источника дополнительного кровоснабжения играли внутренние грудные сосуды. Артериальные анастомозы с правой ободочной артерией сформированы у 41,1\% (7/17) пациентов, со средней ободочной артерией - у 58,9\% (10/17). Дополнительные дренирующие межвенозные анастомозы потребовались 23,5\% (4/17) пациентов, из числа которых у 25\% (1/4) с правой ободочной веной и $75 \%$ (3/4) - со средней ободочной веной.

При анализе непосредственных результатов лечения критериями оценки были продолжительность оперативных вмешательств, объем интраоперационной кровопотери, длительность стационарного лечения, общая частота послеоперационных осложнений и ее структура, частота развития респираторных осложнений, 30- и 60-дневная послеоперационная летальность, частота выполнения реопераций после эзофагоколопластики и ее причины. Также оценивалась частота развития несостоятельности пищеводно-толстокишечного анастомоза и частота некроза толстокишечного трансплантата на 8-е сутки после операции по данным рентгеноскопии с водорастворимым контрастным веществом. Отдаленные результаты лечения оценивали по частоте развития поздних рубцовых стриктур пищеводно-толстокишечного анастомоза спустя 3 месяца после операции по эндоскопическим данным (фиброэзофагоколоноскопия), а также по показателям общей 5-летней выживаемости и медианы выживаемости.

\section{Статистика}

Нормальность распределения признаков оценивали с применением теста КолмогороваСмирнова. Распределение признака считали 
отличающимся от нормального при $\mathrm{p}<0,05$. Изучаемые количественные признаки не подчинялись нормальному распределению, и по этой причине количественные параметры в работе были представлены в виде медианы $(\mathrm{Me})$ и квартилей $(\mathrm{Q})$ - Мe $(\mathrm{Q} 25 ; \mathrm{Q} 75)$.

$\mathrm{C}$ целью определения различий между количественными переменными при отклонении от нормального распределения использовался непараметрический метод Вилкоксона для внутригрупповых зависимых переменных. Различия считали достоверными при $\mathrm{p}<0,05$.

Показатели выживаемости рассчитывали по методу Каплана-Мейера с отображением функции выживаемости в виде ступенчатого графика и указанием на кривой цензурированных наблюдений, к которым относили выживших пациентов. Точкой отсчета являлось время начала хирургического лечения карцином пищевода и пищеводно-желудочного перехода. С использованием таблиц дожития рассчитывались показатели средней продолжительности жизни и медианы выживаемости с указанием стандартной ошибки.

\section{Результаты}

В общей когорте пациентов продолжительность оперативных вмешательств составила $345,0(310,0 ; 407,5)$ минут. Объем интраоперационной кровопотери не превышал 400,0 (300,0; $500,0)$ мл.

Общая частота развития послеоперационных осложнений составила 47,5\% (19/40): нагноение раны $-25 \%(10 / 40)$, пневмония $-7,5 \%$ $(3 / 40)$, кровотечение - 5\% (2/40), перитонит $5 \%(2 / 40)$, тромбоэмболия ветвей легочной артерии $-2,5 \%(1 / 40)$, острая легочно-сердечная недостаточность $-2,5 \%(1 / 40)$.

Несостоятельность пищеводно-толстокишечного анастомоза развилась в $12,5 \%$ (5/40) наблюдений, некроз проксимальных отделов толстокишечного трансплантата - в 5\% (2/40). Средние сроки диагностики вышеуказанных осложнений составили $6(3,0 ; 8,0)$ дней. Поздние рубцовые стриктуры пищеводно-толстокишечного анастомоза сформировались в 7,5\% (3/40) наблюдений.

Средняя продолжительность пребывания пациентов в стационаре составила 27,5 (21,0; $40,5)$ дня. 30- и 60-дневная летальность составила $7,5 \%(3 / 40)$ и $10 \%(4 / 40)$ случаев соответственно. Среди причин, вызвавших летальные исходы, превалировали респираторные осложнения. Так, двусторонняя полисегментарная пневмония составила 75\% (3/4) летальных случаев, некроз трансплантата - у 25\% (1/4).
Общая 5-летняя выживаемость составила $26,9 \%$ (рис. 1).

Медиана выживаемости равнялась 19,4 $\pm 3,6$ (95\% ДИ 12,4-26,4) мес., средняя продолжитель-

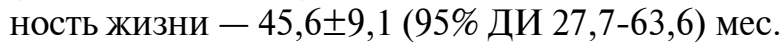

\section{Обсуждение}

Хирургический метод лечения пациентов с карциномами пищевода и пищеводно-желудочного переходя является единственно радикальным, сопряжен с высокой частотой послеоперационных осложнений и летальности. Основными причинами летальности являются легочно-сердечные осложнения, а также осложнения ишемического генеза, к которым относятся несостоятельность пищеводно-органного анастомоза и/или некроз (локальный или тотальный) трансплантата. Вторично развиваются кардиальные (нарушения ритма, сердечная недостаточность), респираторные (полисегментарные пневмонии) и гнойно-септические (медиастинит, эмпиема) осложнения, которые могут явиться причиной послеоперационной летальности.

По данным E.S. Kassis et al., на 7595 эзофагэктомий частота шейных и внутриплевральных несостоятельностей анастомоза составила $12,3 \%$ и 9,3\% наблюдений соответственно [10]. В большинстве случаев лечение данного типа осложнений направлено на сохранение пищеводного анастомоза путем применения различных вариантов дренирования и/или стентирования анастомотической зоны. У части пациентов, у которых развился фокальный или тотальный некроз трансплантата, тактика по сохранению анастомоза в условиях эмпиемы плевры и медиастинита может оказаться непригодной, что может потребовать выполнения разобщающих операций, направленных на ликвидацию септического очага из средостения. По некоторым

Рис. 1. Общая выживаемость пациентов после повторной эзофагоколопластики.

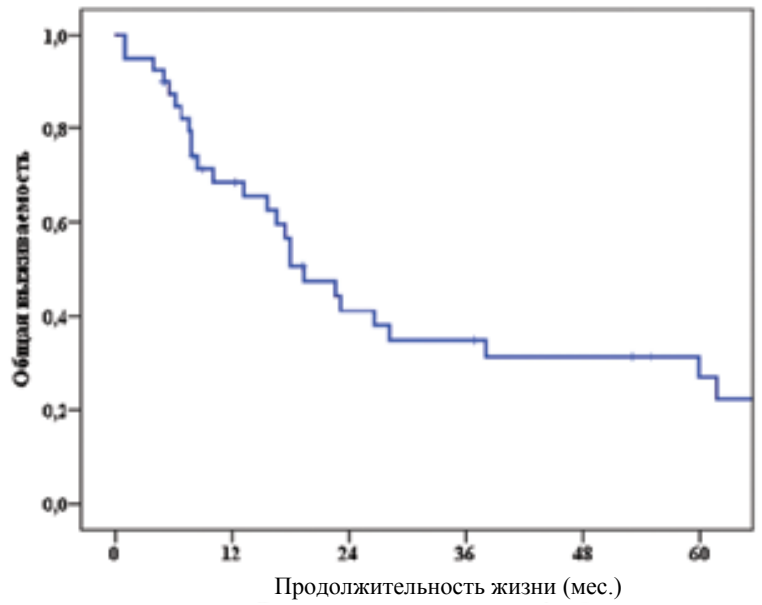


данным, несостоятельности и некрозы трансплантата, требующие ревизионной хирургии, имеют место у $4 \%$ и $2 \%$ оперированных пациентов соответственно [11]. Такая ситуация нередко требует разобщения, которое сопровождается инвалидизацией пациента за счет прерывания естественного пищеварительного пассажа, так как формируются стомы (эзофагостома и гастро- или еюностома).

Использование толстой кишки для пластики пищевода не является рутинно применяемой процедурой из-за потенциально высокого риска развития осложнений и летальности. Формирование, размещение и проведение искусственного пищевода после эзофагэктомии у взрослых онкологических пациентов представляет собой сложную проблему, дискуссия на предмет которой не прекращается и в настоящее время, хотя данный раздел хирургии насчитывает более ста лет. Необходимость использования толстой кишки для эзофагопластики не вызывает сомнений в случаях невозможности использования собственного желудка или тонкой кишки. Более высокая частота развития послеоперационных осложнений после толстокишечной пластики пищевода по сравнению с желудочной пластикой обусловлена продолжительностью и травматичностью вмешательства, необходимостью формирования до 3-4 дигестивных соустий. Следует отметить, что толстая кишка, как кондуит, обладает целым рядом неоспоримых преимуществ: длина, относительная кислотоустойчивость и хорошее кровоснабжение. В настоящее время не существует общепринятого мнения в отношении того, какой вариант толстокишечной пластики пищевода наиболее эффективно применять в повседневной практике. Это касается, прежде всего, фланга кишки (правый, левый или комбинированный вариант), позиции трансплантата (изо- или антиперистальтическая), пути проведения трансплантата (загрудинно, в заднем средостении или подкожно) к месту формирования анастомоза с пищеводом (шея или плевральная полость). Зачастую тип применяемого оперативного вмешательства по созданию искусственного пищевода из собственной толстой кишки зависит от собственных индивидуальных предпочтений и опыта клиники.

Отдельную категорию представляют онкологические пациенты после осложнений первичной пластики пищевода собственным желудком или тонкой кишкой (некроз трансплантата и/или несостоятельность анастомоза), потребовавших выполнения разобщающих операций. Данное обстоятельство приводит к невозможности повторного использования их в качестве пластического материала для формирования искусственного пищевода. Эзофагопластика в таких ситуациях представляет собой крайне трудную проблему онкохирургии, нередко требующую применения нестандартных вариантов реконструкции пищевода из имеющегося в наличии пластического резерва в виде толстой кишки.

Так, наибольший опыт выполнения повторных толстокишечных пластик пищевода по данным зарубежной литературы, имеется у V. Reslinger et al., которые привели 18 повторных реконструкций пищевода, 15 из которых были выполнены после ранее развившихся некрозов перемещенного желудка [3].

K.A. Kesler et al. приводят опыт 5 повторных реконструктивных операций васкуляризированным трансплантатом из толстой кишки, выполненных отдельным хирургическим этапом, после неудавшейся попытки пластики удаленного пищевода желудочным трансплантатом, так как развился некроз последнего [2].

Ключевым моментом успешности выполнения повторной пластики пищевода является направление усилий на предотвращение натяжения на проксимальный анастомоз пищевода с толстокишечным трансплантатом путем выкраивания кишечного сегмента с достаточным кровоснабжением и необходимой длины. Это позволяет обеспечить профилактику нарушений кровоснабжения трансплантата, а также снизить частоту развития несостоятельности анастомоза ишемического генеза и/или некроза трансплантата. Эти осложнения относятся к наиболее опасным, так как могут вызвать септическое состояние пациента и нередко являются одной из причин послеоперационной летальности.

Множество технических аспектов, касающихся выбора толстокишечного сегмента, пути проведения, позиции трансплантата, варианта формирования анастомоза с пищеводом, могут оказывать значимое влияние как на непосредственные, так и на отдаленные результаты лечения. В связи с этим указанный тип вмешательства осуществляется, как правило, на основе авторских предпочтений и собственного опыта некоторых хирургических школ.

Факторами, осложняющими выполнение повторной реконструкции пищевода, являются выраженные спаечные процессы в брюшной полости после предшествующей операции (эзофагэктомия или резекция пищевода с первичной пластикой и последующая разобщающая операция). Спаечные процессы нередко затрагивают как верхний, так и нижний этажи брюшной полости и зачастую не позволяют четко визуализировать брыжеечные сосуды толстой кишки, что 
в значительной мере затрудняет мобилизацию выбранного для пластики кишечного сегмента. Кроме того, наличие ранее сформированной гастро- или еюностомы в левой мезогастральной области отягощает мобилизацию селезеночного изгиба и левого фланга толстой кишки.

Вместе с тем, повторная пластика характеризуется значительным дефицитом пластического материала, так как желудок или тонкая кишка были использованы для первичной пластики пищевода, а также для формирования гастро- или еюностомы. При такой ситуации единственным пластическим резервом остается собственная толстая кишка.

Техническая сложность и ожидаемая продолжительность вмешательства должны учитываться при выборе толстокишечного сегмента для эзофагопластики. Решение об использовании для реконструкции правого или левого фланга должно быть взвешенным и базироваться на оценке индивидуальных особенностей кровоснабжения толстой кишки, учете длины формируемого толстокишечного трансплантата, достаточного для отдаленного перемещения в загрудинном предфасциальном канале на шею.

Выкраивание толстокишечного сегмента из правого фланга после ранее перенесенной разобщающей операции требует меньше травматичных манипуляций, технически проще, сопровождается меньшей продолжительностью и объемом кровопотери, так как позволяет избежать тотальной мобилизации толстой кишки (мобилизация выполняется субтотально до селезеночного изгиба). В свою очередь, формирование трансплантата из левого фланга толстой кишки требует мобилизации селезеночного изгиба, который зачастую находится в спаечном процессе (риск повреждения связи между бассейнами брыжеечных артерий), а также нисходящей ободочной кишки, мобилизацию которой затрудняет ранее сформированная гастро- или еюностома. Таким образом, эзофагопластика левым флангом толстой кишки требует тотальной ее мобилизации, так как только при этом условии возникает возможность сформировать анастомоз без натяжения между оставшимися сегментами толстой кишки.

Эзофагопластика толстокишечным трансплантатом в изоперистальтической позиции является более физиологичной, чем в антиперистальтической позиции. Это обусловлено сохранением перистальтической активности в отдаленные сроки. Трансплантат из левой половины толстой кишки, как правило, удается расположить именно в антиперистальтической позиции, что зачастую является причиной рефлюкса желчи и требует постановки на- зоинтестинального зонда для профилактики рефлюкс-эзофагита и в последующем рубцовых стриктур анастомоза. Однако положительным моментом является меньший диаметр и более устойчивое кровоснабжение такого кишечного сегмента, который будет обладать меньшим риском развития избыточности, свойственной трансплантатам из правого фланга.

При повторной пластике для проведения трансплантата на шею заднее средостение зачастую оказывается непригодным в связи с предшествующими вмешательствами. Поэтому предпочтение отдается в пользу ретростернального расположения трансплантата, которое обеспечивает защиту от травматизации. При образовании загрудинного тоннеля существует риск повреждения плевры со вскрытием одной или двух плевральных полостей и последующим проваливанием туда трансплантата, перегибом и ротацией по оси с возможным нарушением кровоснабжения вплоть до некроза. Также одним из потенциальных факторов, приводящих к нарушению кровоснабжения толстокишечного сегмента, может быть сдавление в канале верхней апертуры грудной клетки (на уровне рукоятки грудины в области выхода на шею). Это может быть причиной дисфагии с нарушением проходимости по искусственному пищеводу.

Разнородность представленных в литературе данных, касающихся вопросов толстокишечной пластики пищевода, зачастую не позволяет сравнивать результаты опыта различных клиник по частоте послеоперационных осложнений и летальности. Результаты, как правило, сильно различаются в зависимости от опыта различных хирургических школ, а также от характера реконструкции (первичная, повторная или отсроченная).

Taк, C.D. Klink et al. приводят показатель частоты некроза колотрансплантата, который достигает 9,3\% (4/43) [12]. По данным V. Reslinger et al., имеющих наибольший опыт среди зарубежных авторов по проблемам повторной эзофагоколопластики $(\mathrm{n}=18)$, частота некроза толстокишечного трансплантата составила 16,7\% (3/18). В общей когорте оперированных ими пациентов данный показатель равнялся $14,3 \%$ (4/28) [3]. В данной работе частота некроза проксимальных сегментов колотрансплантата составила 5\% (2/40) случаев вследствие сдавления рукояткой грудины при проведении на шею. Для профилактики компрессии трансплантата в канале верхней апертуры грудной клетки в последующем выполнялось рассечение группы передних прямых мышц шеи слева, что позволяло расширить загрудинное пространство в области выхода на шею [8]. 
Несостоятельность пищеводно-толстокишечного анастомоза на шее встречается у $30,2-35,7 \%$ оперированных пациентов [3, 6, 12]. Процент во многом зависит от опыта клиники, объема выполняемых вмешательств данного типа, числа представленных наблюдений. Разбежка в показателях объясняется тем, что многие авторы включают в анализ клинически незначимые несостоятельности анастомозов, диагностируемые только рентгенологически в виде затека контрастного вещества без какойлибо клинической картины. В представленной работе частота несостоятельности шейного пишеводно-толстокишечного анастомоза составила 12,5\% (5/40) наблюдений. Все несостоятельности, не сопровождавшиеся некрозом проксимальных сегментов колотрансплантата, лечились консервативно путем санации и дренирования шейной раны в зоне осложнения. Это приводило к спонтанному заживлению, которое наступало в сроки до 7-21 дня от начала лечения.

Некоторые авторы указывают на эффективность применения васкуляризации (сосудистого усиления) толстокишечного трансплантата с целью профилактики развития ишемических осложнений (несостоятельности и/или некроза). Указанный подход в ряде случаев позволяет обеспечить достаточное кровоснабжение выкроенного кишечного сегмента в условиях измененной сосудистой анатомии толстой кишки $[2,6,7,8,9]$. Васкуляризация трансплантата применена у 42,5\% (17/40) пациентов. [8]. Общая схема представлена на рис. 2.

Выполнение повторных реконструкций пищевода после осложнений первичной пластики пищевода желудком или тонкой кишкой не всегда сопровождается необходимостью в сосудистом усилении. Показанием к васкуляризации в работе было наличие перерывов краевого (маргинального или пристеночного) питающего сосуда по правому или левому флангу толстой кишки, а также перерыв анастомотического сосуда между бассейнами верхней и нижней брыжеечных артерий [8]. Указанные особенности могут быть диагностированы на дооперационном этапе путем выполнения селективной ангиографии - верхней и нижней мезентерикографии. Это позволяет планировать уровни пересечения питающих сосудов для формирования трансплантата, наиболее подходящего в данных анатомических условиях (рис. 3).

Послеоперационная летальность после эзофагоколопластики может достигать 9-17\% $[2,3,12]$. По данным исследования R.A. Fisher et al., включившего все толстокишечные пластики, выполненные за десятилетний период в Великобритании, послеоперационная летальность составила $11 \%$ (35/318) наблюдений [5]. J. Brown et al. приводит данные мета-анализа 1849 толстокишечных пластик пищевода из 27 различных исследований, где показатель послеоперационной летальности для всей когорты пациентов варьировал в зависимости от фланга толстой кишки, использованного для реконструкции. Так, для эзофагопластики с использованием правого и левого флангов толстой кишки послеоперационная летальность составила $10,1 \%$ и $6,5 \%$ соответственно [13].

При анализе собственного материала показатели 30- и 60-дневной послеоперационной летальности составили 7,5\% (3/40) и 10\% (4/40) соответственно. Следует отметить, что большинство авторов в своих публикациях четко не

Рис. 2. Схема повторной загрудинной толстокишечной пластики пищевода после разобщаюей операции

по поводу осложнений первичной реконструкции пищевода желудком: вид сбоку (А) и вид спереди (Б).

1 - пищеводно-толстокишечный анастомоз, 2 - межартериальный сосудистый анастомоз между внутренней грудной и ободочно-кишечной артерией, 3 - толсто-тонкокишечный анастомоз, 4 - желудочный трансплантат, резецированный в ходе разобщающей операции.
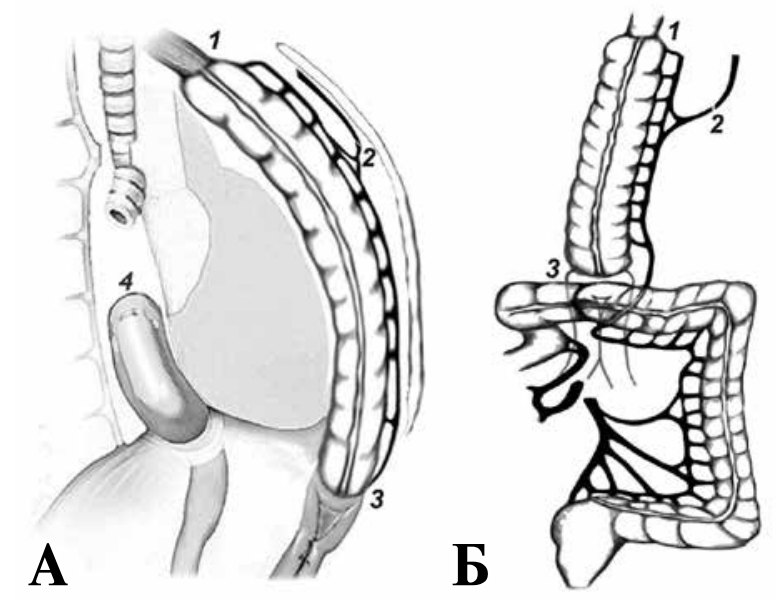

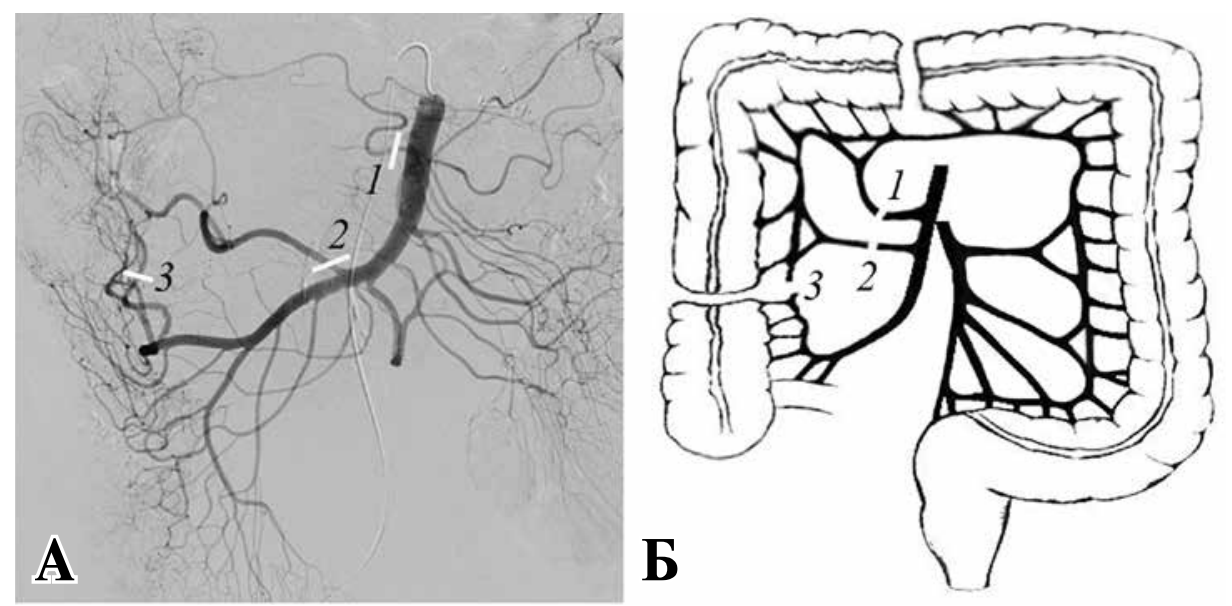

Рис. 3. Схема дооперационного планирования уровня пересечения питающих сосудов: верхняя мезентерикография (А) и схема формирования трансплантата (Б).

1 - пересечение средней ободочной артерии, 2 - пересечение правой ободочной артерии,

3 - пересечение краевого (пристеночного или маргинального) сосуда по правому флангу толстой кишки.

разграничивают показатели послеоперационной летальности (госпитальная, 30-, 60-дневная), что и имеет ключевое значение при сравнительном анализе. По этой причине довольно трудно сравнивать между собой различные исследования, в том числе и из-за неполноты представляемых данных.

Одним из показателей, определяющих эффективное функционирование пересаженного толстокишечного сегмента, является частота развития поздних рубцовых стриктур шейного пищеводно-толстокишечного анастомоза. Рубцевание анастомоза встречается у 18,6-32,1\% пациентов, перенесших толстокишечную эзофагопластику [3, 12]. В данной работе частота стриктурообразования зарегистрирована у 7,5\% (3/40) пациентов по данным фиброэзофагоколоноскопии, которая выполнялась на 3-й месяц после эзофагоколопластики. Развитие рубцовой стриктуры было обусловлено вторичным заживлением (через шейный свищ) анастомозов, осложненных несостоятельностью.

Экстренные реоперации по причинам развития хирургических осложнений (некроза, несостоятельности, непроходимости) являются нередкими и достигают 14,2-32,1\% после эзофагоколопластики [3, 13, 14]. Необходимость в выполнении реопераций в данной работе возникла в 7,5\% (3/40) случаев. В 5\% (2/40) наблюдений потребовалась разобщающая операция с удалением загрудинно расположенного толстокишечного трансплантата по поводу некроза проксимального его сегмента, а в 2,5\% (1/40) разобщение межтолстокишечного анастомоза по поводу несостоятельности последнего с формированием концевой асцендостомы.

Развитие респираторных осложнений (респираторный дистресс-синдром взрослых и пневмония) является одной из частых причин послеоперационной смертности у данной категории пациентов и встречается в 35-42,8\% случаев $[3,7,15]$. В данной работе из общего числа пациентов $(\mathrm{n}=40)$ пневмония диагностирована у $12,5 \%(5 / 40)$ человек, из которых 7,5\% (3/40) пациентов умерло по данной причине в сроки до 60 суток после вмешательства.

Естественный пероральный прием пищи был восстановлен у 95\% (38/40) пациентов. В $5 \%$ (2/40) случаев было выполнено удаление толстокишечного кондуита по причине некроза проксимальной его трети. В литературе приводится данный показатель в пределах 75-80\% случаев [14]. Нефункциональность трансплантата зачастую обусловлена рубцовыми стриктурами анастомоза, избыточностью трансплантата с возможностью его перекрута по оси и развитием стриктуры на протяжении.

Важным преимуществом загрудинного пути проведения является изоляция трансплантата от переднего средостения и плевральных полостей за счет расположения в созданном предфасциальном пространстве. Отграничивающую роль выполняют внутригрудная фасция и плевральные мешки. По этой причине при формировании загрудинного предфасциального тоннеля необходимо минимизировать риск вскрытия плевральных полостей.

Развитие ишемических осложнений после радикальных операций по поводу карцином пищевода и пищеводно-желудочного перехода сопровождается высокой летальностью. Активная тактика лечения позволяет спасти пациента ценой выполнения разобщающей операции со стомированием [16]. Повторная толстокишечная пластика пищевода является «спасительной» операцией, позволяющей вос- 
становить непрерывность пищеварительного тракта при невозможности использования для пластики желудка или тонкой кишки. Выполнение повторной реконструкции создает условия для долговременной выживаемости за счет улучшения качества жизни. Полученный показатель 5-летней выживаемости $(26,8 \%)$ соотносится с данными по выживаемости после стандартных операций, где искомый показатель составляет 25,4\% [17].

Таким образом, индивидуализированный подход при выборе варианта эзофагопластики в условиях дефицита пластического материала несет в себе потенциал долговременной выживаемости за счет снижения частоты развития послеоперационных осложнений и улучшения качества жизни пациентов с карциномами пищевода и пищеводно-желудочного перехода.

\section{Заключение}

При повторной толстокишечной пластике пищевода использование правого фланга толстой кишки в изоперистальтической позиции является предпочтительным, так как позволяет сформировать толстокишечный сегмент по физиологическому направлению перистальтики путем менее травматичной субтотальной мобилизации ободочной кишки, минимизируя риск повреждения анастомотической связи между бассейнами верхней и нижней брыжеечных артерий.

Формирование жизнеспособного толстокишечного трансплантата при повторной эзофагоколопластике должно проводиться с учетом вариабельности ангиоархитектоники ободочной кишки, что требует дооперационного планирования уровня пересечения питающих сосудов на основании данных, полученных при селективной ангиографии (верхней и нижней мезентерикографии).

Повторная толстокишечная пластика пищевода при хирургическом лечении карцином пищевода и пищеводно-желудочного перехода является «спасительной» процедурой для пациентов, перенесших разобщающие (стомирующие) оперативные вмешательства, позволяющей повысить качество жизни онкологических пациентов за счет восстановления естественного пищеварительного пассажа и добиться удовлетворительных непосредственных и отдаленных результатов лечения.

\section{Финансирование}

Работа выполнялась в соответствии с грантом Президента Республики Беларусь в здравоохранении на 2018 год.

\section{Конфликт интересов}

Автор заявляет, что конфликт интересов отсутствует.

\section{Одобрение комитета по этике}

Одобрено комитетом по этике Республиканского научно-практического центра онкологии и медицинской радиологии им. Н.Н. Александрова.

\section{ЛИТЕРАТУРА}

1. Vijay K, Godara R, Vijayvergia V. Failed Gastric Pull up after Esophagectomy Managed by Colonic Interposition. Indian J Surg. 2013 Jun;75(Suppl 1):34749. doi: $10.1007 / \mathrm{s} 12262-012-0662-\mathrm{X}$

2. Kesler KA, Pillai ST, Birdas TJ, Rieger KM, Okereke IC, Ceppa D, Socas J, Starnes SL. "Supercharged" isoperistaltic colon interposition for long-segment esophageal reconstruction. Ann Thorac Surg. 2013 Apr;95(4):1162-68; discussion 1168-69. doi: 10.1016/j. athoracsur.2013.01.006

3. Reslinger V, Tranchart H, D'Annunzio E, Poghosyan T, Quero L, Munoz-Bongrand N, Corte H, Sarfati E, Cattan P, Chirica M. Esophageal reconstruction by colon interposition after esophagectomy for cancer analysis of current indications, operative outcomes, and longterm survival. J Surg Oncol. 2016 Feb;113(2):15964. doi: $10.1002 /$ jso. 24118

4. Sacak B, Orfaniotis G, Nicoli F, Liu EW, Ciudad $\mathrm{P}$, Chen $\mathrm{SH}$, Chen HC. Back-up procedures following complicated gastric pull-up procedure for esophageal reconstruction: Salvage with intestinal flaps. Microsurgery. 2016 Oct;36(7):567-72. doi: 10.1002/micr.22520

5. Fisher RA, Griffiths EA, Evison F, Mason RC, Zylstra J, Davies AR, Alderson D, Gossage JA. A national audit of colonic interposition for esophageal replacement. Dis Esophagus. 2017 May 1;30(5):1-10. doi: 10.1093/dote/dow003

6. Awsakulsutthi S, Havanond C. A retrospective study of anastomotic leakage between patients with and without vascular enhancement of esophageal reconstructions with colon interposition: Thammasat University Hospital experience. Asian J Surg. 2015 Jul;38(3):14549. doi: 10.1016/j.asjsur.2015.01.005

7. Uchiyama H, Shirabe K, Morita M, Kakeji Y, Taketomi A, Soejima Y, Yoshizumi T, Ikegami T, Harada N, Kayashima H, Morita K, Maehara Y. Expanding the applications of microvascular surgical techniques to digestive surgeries: a technical review. Surg Today. 2012 Jan;42(2):111-20. doi: 10.1007/ s00595-011-0032-5

8. Ильин ИА, Малькевич ВТ. Повторная и отсроченная эзофагопластика в лечении карцином пищевода и пищеводно-желудочного перехода. Изв НАН Беларуси. Сер мед наук. 2016;(2):15-22.

9. Saeki H, Morita M, Harada N, Egashira A, Oki E, Uchiyama H, Ohga T, Kakeji Y, Sakaguchi Y, Maehara Y. Esophageal replacement by colon interposition with microvascular surgery for patients with thoracic esophageal cancer: the utility of superdrainage. Dis Esophagus. 2013 Jan;26(1):50-56. doi: 10.1111/j.14422050.2012.01327.x

10. Kassis ES, Kosinski AS, Ross P Jr, Koppes KE, Donahue JM, Daniel VC. Predictors of anastomotic 
leak after esophagectomy: an analysis of the society of thoracic surgeons general thoracic database. Ann Thorac Surg. 2013 Dec;96(6):1919-26. doi: 10.1016/j.athoracsur.2013.07.119

11. Luketich JD, Pennathur A, Awais O, Levy RM, Keeley S, Shende M, Christie NA, Weksler B, Landreneau RJ, Abbas G, Schuchert MJ, Nason KS. Outcomes after minimally invasive esophagectomy: review of over 1000 patients. Ann Surg. 2012 Jul;256(1):95103. doi: 10.1097/SLA.0b013e3182590603

12. Klink CD, Binnebösel M, Schneider M, Ophoff K, SchumpelickV, Jansen M. Operative outcome of colon interposition in the treatment of esophageal cancer: a 20-year experience. Surgery. 2010 Apr;147(4):491-6. doi: 10.1016/j.surg.2009.10.045

13. Brown J, Lewis WG, Foliaki A, Clark GWB, Blackshaw GRJC, Chan DSY. Colonic Interposition After Adult Oesophagectomy: Systematic Review and Meta-analysis of Conduit Choice and Outcome. J Gastrointest Surg. 2018 Jun;22(6):1104-11. doi: 10.1007/ s11605-018-3735-8

14. Bakshi A, Sugarbaker DJ, Burt BM. Alternative conduits for esophageal replacement. Ann Cardiothorac Surg. 2017 Mar;6(2):137-43. doi: 10.21037/ acs.2017.03.07

15. Ceroni M, Norero E, Henríquez JP, Vicuela E, Briceco E, Martínez C, Aguayo G, Araos F, González P, Díaz A, Caracci M. Total esophagogastrectomy plus extended lymphadenectomy with transverse colon interposition: a treatment for extensive esophagogastric junction cancer. World J Hepatol. $2015 \mathrm{Oct}$ 8;7(22):2411-17. doi: 10.4254/wjh.v7.i22.2411

16. Малькевич ВТ, Жарков ВВ, Оситрова ЛИ, Курчин ВП, Баранов АЮ, Ильин ИА. Новые подходы к лечению внутриплевральных осложнений в хирургии рака пищевода. Новости Хирургии. 2012;20(3):74-80. http://www.surgery.by/pdf/full text/2012_3_12_ft.pdf

17. Океанов АЕ, Моисеев ПИ, Левин ЛФ, Евмененко АА, Суконко ОГ. (ред). Статистика онкологических заболеваний в Республике Беларусь $(2007-2016)=$ Statistics of cancer diseases in the Republic of Belarus (2007-2016): аналитический обзор по данным Белорусского канцер-регистра. Минск, РБ: РНПЦ ОМР; 2017. 286 с.

\section{REFERENCES}

1. Vijay K, Godara R, Vijayvergia V. Failed Gastric Pull up after Esophagectomy Managed by Colonic Interposition. Indian J Surg. 2013 Jun;75(Suppl 1):34749. doi: $10.1007 / \mathrm{s} 12262-012-0662-\mathrm{X}$

2. Kesler KA, Pillai ST, Birdas TJ, Rieger KM, Okereke IC, Ceppa D, Socas J, Starnes SL. "Supercharged" isoperistaltic colon interposition for long-segment esophageal reconstruction. Ann Thorac Surg. 2013 Apr;95(4):1162-68; discussion 1168-9. doi: 10.1016/j. athoracsur.2013.01.006

3. Reslinger V, Tranchart H, D'Annunzio E, Poghosyan T, Quero L, Munoz-Bongrand N, Corte H, Sarfati E, Cattan P, Chirica M. Esophageal reconstruction by colon interposition after esophagectomy for cancer analysis of current indications, operative outcomes, and longterm survival. J Surg Oncol. 2016 Feb;113(2):15964. doi: $10.1002 /$ jso. 24118

4. Sacak B, Orfaniotis G, Nicoli F, Liu EW, Ciudad $\mathrm{P}$, Chen SH, Chen HC. Back-up procedures following complicated gastric pull-up procedure for esophageal reconstruction: Salvage with intestinal flaps. Microsurgery. 2016 Oct;36(7):567-72. doi: 10.1002/micr.22520 5. Fisher RA, Griffiths EA, Evison F, Mason RC, Zylstra J, Davies AR, Alderson D, Gossage JA. A national audit of colonic interposition for esophageal replacement. Dis Esophagus. 2017 May 1;30(5):1-10. doi: $10.1093 /$ dote/dow003

6. Awsakulsutthi S, Havanond C. A retrospective study of anastomotic leakage between patients with and without vascular enhancement of esophageal reconstructions with colon interposition: Thammasat University Hospital experience. Asian J Surg. 2015 Jul;38(3):14549. doi: 10.1016/j.asjsur.2015.01.005

7. Uchiyama H, Shirabe K, Morita M, Kakeji Y, Taketomi A, Soejima Y, Yoshizumi T, Ikegami T, Harada N, Kayashima H, Morita K, Maehara Y. Expanding the applications of microvascular surgical techniques to digestive surgeries: a technical review. Surg Today. 2012 Jan;42(2):111-20. doi: 10.1007/ s00595-011-0032-5

8. Ilyin IA, Malkevich VT. Repeated and delayed esophagoplasty in esophageal and gastroesophageal cancer treatment. Izv NAN Belarusi. Ser Med Nauk. 2016;(2):15-22. (in Russ.)

9. Saeki H, Morita M, Harada N, Egashira A, Oki E, Uchiyama H, Ohga T, Kakeji Y, Sakaguchi Y, Maehara Y. Esophageal replacement by colon interposition with microvascular surgery for patients with thoracic esophageal cancer: the utility of superdrainage. Dis Esophagus. 2013 Jan;26(1):50-56. doi: 10.1111/j.14422050.2012.01327.x

10. Kassis ES, Kosinski AS, Ross P Jr, Koppes KE, Donahue JM, Daniel VC. Predictors of anastomotic leak after esophagectomy: an analysis of the society of thoracic surgeons general thoracic database. Ann Thorac Surg. 2013 Dec;96(6):1919-26. doi: 10.1016/j.athoracsur.2013.07.119

11. Luketich JD, Pennathur A, Awais O, Levy RM, Keeley S, Shende M, Christie NA, Weksler B, Landreneau RJ, Abbas G, Schuchert MJ, Nason KS. Outcomes after minimally invasive esophagectomy: review of over 1000 patients. Ann Surg. 2012 Jul;256(1):95103. doi: 10.1097/SLA.0b013e3182590603

12. Klink CD, Binnebösel M, Schneider M, Ophoff K, SchumpelickV, Jansen M. Operative outcome of colon interposition in the treatment of esophageal cancer: a 20-year experience. Surgery. 2010 Apr;147(4):491-96. doi: 10.1016/j.surg.2009.10.045

13. Brown J, Lewis WG, Foliaki A, Clark GWB, Blackshaw GRJC, Chan DSY. Colonic Interposition After Adult Oesophagectomy: Systematic Review and Meta-analysis of Conduit Choice and Outcome. J Gastrointest Surg. 2018 Jun;22(6):1104-11. doi: 10.1007/ s11605-018-3735-8

14. Bakshi A, Sugarbaker DJ, Burt BM. Alternative conduits for esophageal replacement. Ann Cardiothorac Surg. 2017 Mar;6(2):137-43. doi: 10.21037/ acs.2017.03.07

15. Ceroni M, Norero E, Henríquez JP, Vicuela E, Briceco E, Martínez C, Aguayo G, Araos F, González P, Díaz A, Caracci M. Total esophagogastrectomy plus extended lymphadenectomy with transverse colon interposition: a treatment for extensive esophagogastric junction cancer. World J Hepatol. 2015 Oct 8;7(22):2411-17. doi: 10.4254/wjh.v7.i22.2411

16. Malkevich VT, Zharkov VV, Ositrova LI, Kurchin VP, Baranov AYu, Ilyin I.A. New approaches to treatment of intrapleural complications in esophageal 
cancer surgery. Novosti Khirurgii. 2012;20(3):74-80. http://www.surgery.by/pdf/full_text/2012_3_12_ft.pdf (in Russ.)

17. Okeanov AE, Moiseev PI, Levin LF, Evmenenko AA, Sukonko OG. (red). Statistika onkologicheskikh

\section{Адрес для корреспонденции}

223040, Республика Беларусь,

Минская область, Минский район, агрогородок Лесной 2,

Республиканский научно-практический центр онкологии и медицинской радиологии им. Н.Н. Александрова, хирургический отдел, тел. раб.: 801738995 32, e-mail: ileus@tut.by, Ильин Илья Анатольевич

\section{Сведения об авторах}

Ильин Илья Анатольевич, к.м.н., ведущий научный сотрудник хирургического отдела, государственное учреждение «Республиканский научно-практический центр онкологии и медицинской радиологии им. Н.Н. Александрова», Минская область, Минский район, агрогородок Лесной 2, Республика Беларусь.

http://orcid.org/0000-0002-5314-7618

\section{Информация о статье}

Поступила 31 мая 2018 г.

Принята к публикации 11 февраля 2019 г. Доступна на сайте 30 апреля 2019 г. zabolevanii v Respublike Belarus' (2007-2016)=Statistics of cancer diseases in the Republic of Belarus (20072016): analiticheskii obzor po dannym Belorusskogo kantser-registra. Minsk, RB: RNPTs OMR; 2017. 286 p. (in Russ.)

\section{Address for correspondence}

223040, The Republic of Belarus, Minsk region, Lesnoy 2,

N.N. Alexandrov National Cancer

Centre of Belarus, Surgical Department.

Tel. office.: 801738995 32,

e-mail: ileus@tut.by,

Ilya A. Ilyin

\section{Information about the authors}

Ilyin Ilya A., PhD, Leading Researcher of the Surgical Department, N.N. Alexandrov National Cancer Centre of Belarus, Minsk, Republic of Belarus.

http://orcid.org/0000-0002-5314-7618

\section{Article history}

Arrived 31 May 2018

Accepted for publication 11 February 2019

Available online 30 April 2019 